\title{
Evaluación en FLIP-Teaching: una alternativa a los exámenes parciales. Evaluación continua mediante tareas y casos.
}

Juan Vte. Oltra Gutiérrez ${ }^{a}$

${ }^{a}$ Universitat Politécnica de Valencia, Departamento de Organización de Empresas (jvoltra@omp.upv.es)

\begin{abstract}
The FLIP teaching experience have many challenges, one of the most unique is evaluation. Faced with the current that defends to maintain the traditional in a way that remains the same as the rest of groups with face-to-face teaching, in the present work an experience of continuous evaluation is presented that allows to use for this the time of classroom released by the classroom teaching that is has taken out of it
\end{abstract}

Keywords: FLIP-Teaching, continuous evaluation, classroom cases, work outside the classroom, methodology.

\begin{abstract}
Resumen
La experiencia FLIP teaching plantea muchos retos, uno de los más singulares es la evaluación. Frente a la corriente que defiende mantener la tradicional de forma que permanezca igual que el resto de grupos con docencia presencial, en el presente trabajo se presenta una experiencia de evaluación continua que permite utilizar para ello el tiempo de aula liberado por la docencia presencial que se ha sacado de la misma
\end{abstract}

Palabras clave: FLIP-Teaching, evaluación continua, casos de aula, trabajo fuera del aula, metodología.

\section{Introducción}

La introducción del modelo de docencia inversa o FLIP Teaching en la Universitat Politècnica de València supuso si no una revolución si al menos un pequeño seísmo entre los profesores implicados. Múltiples prismas, desde aquellos que miraban con recelo la experiencia a quienes se mostraron partidarios de la misma, campos de aplicación tan diversos como son las materias en las que se forman los alumnos de nuestra universidad (de la matemática al derecho pasando por la programación)...

Esta diversidad de origen produjo que convivieran, y conviven, ejecuciones de la experiencia que poco más que el nombre comparten. Prueba de ello son los testimonios de los alumnos en las distintas jornadas de docencia Inversa que se han convocado desde el origen de la experiencia. Podríamos decir, dibujando con trazo grueso, que poco más que el uso de vídeos y el empleo de tiempo en casa, son los elementos comunes. Y aun en algún caso, particularmente me permitiría dudar de ello. 
Uno de los elementos que más controversia genera cuando se organizan reuniones, formales o informales, entre docentes que aplican FLIP-Teaching es el de la evaluación. Sobre una plasmación de ella versa el presente trabajo

\section{Antecedentes}

La asignatura sobre la que se efectúa la presente experiencia es Deontología y Profesionalismo, de segundo semestre en el segundo curso del grado de informática, impartido en la Escuela Técnica Superior de Ingeniería Informática, con un peso de 4,5 créditos (tres créditos correspondientes a teoría, y uno y medio de prácticas)

Cuando hace tres años nos enfrentamos al reto de transformar uno de sus grupos al formato FLIP-Teaching, el primer paso que se dio fue el de localizar información que diera pistas sobre las mejores prácticas, las formas de actuar más adecuadas, además de la formación que el Instituto de Ciencias de la Educación nos facilitó a los docentes implicados.

Así, tras largas lecturas (BENNETT, 2011), (BETHANY, 2012), (MCKEACHIE, 2014), (MARTINEZ, 2014), (MOWAFY, 2013), (RIZZO, 2015), (ROSHAN, 2011), (SAMS, 2013), (SPENCER, 2011), (VASILEVA-STOJANOVSKA, 2015), se conformó el modelo. Para una visión detallada del modelo de partida y las correcciones que en el siguiente curso se aplicaron, pueden consultarse nuestros trabajos (OLTRA, 2015) y (OLTRA, 2016), que sirven de precedente para la presente comunicación.

\section{Evaluación}

Si bien cualquier aspecto del diseño de una experiencia docente en general y en FLIP en particular está sujeto a discrepancias de todo tipo y grado, al ser la evaluación el aspecto que ofrece más datos cuantificables, y por tanto medibles, es la estrella en las discusiones en todo tipo de foros, formales o informales.

La pregunta principal que se suele plantear es: ¿aplicamos a todos los alumnos la misma evaluación? Es una pregunta delicada porque, por una parte, se enarbola la idea de que todos tienen que tener la misma igualdad de oportunidades, y por tanto, las pruebas, sean exámenes parciales o cualquier otra, deben ser idénticas o al menos muy parecidas para todos. Por otra parte, sin embargo, se plantea a modo de respuesta otra pregunta: ¿Podemos aplicar una innovación docente despreciando su ventaja más evidente? La respuesta que se da en la presente comunicación es un NO rotundo. La mayor ventaja de la clase inversa es permitir usar el tiempo de aula para cosas distintas a la exposición magistral, en concreto para la realización de trabajos, debates... Pues bien, si los hacemos, el paso que de forma obvia sigue es evaluarlo. Asignarles nota y que sirvan tanto al alumno para ir avanzando por la asignatura como al profesor para poder tomar la temperatura a su clase en todo momento, y no solo cuando llegan los parciales.

Particularmente siempre he detectado una trampa en esa afirmación, pues desde el momento en que reciben de forma distinta la docencia, de modo que no hay clase magistral en muchos casos, ya estamos alterando la premisa de partida: todos no tienen ya igualdad de oportunidades. Unos tiene la cercanía del profesor que otros, en cambio pierden, pero

(c) EY-NC-ND 2017, UniversitatPolitècnica de València 
disponen de la posibilidad de ver o leer una y otra vez el material que lo sustituye, además de por supuesto acudir a las sesiones de tutoría como todos.

Pero es que aun hay más: si sacamos a la docencia tradicional, a la clase magistral del aula, porque la estamos, permítaseme la expresión, enlatando para su consumo fuera de ella, y las clases las dedicamos a afianzar la teoría mediante ejercicios, test, casos, preguntas del minuto, rol... ¿tiene sentido entonces hacer trabajar a los alumnos de modo que podemos ir comprobando sus avances y no aprovechar esto para alcanzar esa meta que parecía tan utópica y a fuerza de ser empleada vacuamente queda manida y ajada, relegada a buenos propósitos o directamente a eufemismos que cubren una mentira conocida en la guía docente?

Con la idea de partida fija en conseguir este extremo, año a año, se han ido efectuando correcciones hasta llegar a lo que, hoy por hoy, consideramos una versión estable para la evaluación de los grupos FLIP de la asignatura (grupos, pues desde el curso anterior, al grupo piloto sumamos otro grupo, "robándolo" de la docencia convencional)

Para desarrollar el esquema de este modelo de evaluación partiremos de la descripción del modelo convencional: el cómo se evalúa la asignatura en los grupos no FLIP, para así poder establecer las necesarias comparaciones al mostrar el modelo seguido.

\subsection{Evaluación de los grupos "No-Flip"}

Podemos ver un desglose en la tabla siguiente:

Tabla 1. Evaluaciones en grupos convencionales

\begin{tabular}{|l|l|l|l|}
\hline Corresponde a & Tipo & Actos & Peso \\
\hline Práctica & Trabajo académico & 4 & $30 \%(1+1+0,5+0,5)$ \\
\hline Práctica & Coevaluación & 1 & $5 \%(0,5)$ \\
\hline Teoría & Caso & 3 & $15 \%(0,5+0,5+0,5)$ \\
\hline Teoría & $\begin{array}{l}\text { Examen (test } \\
\text { desarrollo) }\end{array}$ & 2 & $\begin{array}{l}50 \%(2,5+2,5) \\
(\text { cada examen: test, } \\
1 \text { punto, desarrollo } \\
1,5 \text { puntos })\end{array}$ \\
\hline
\end{tabular}

Además hay que incluir las actividades de gamificación y extras de la asignatura, que permiten de algún modo "pre-recuperar" partes de la misma, como por ejemplo un pequeño trabajo sobre una jornada con invitados del mundo profesional que cada año se celebra.

Sobre la evaluación en grupos "NO-FLIP" vemos que nada escapa a lo convencional: desde las prácticas diseñadas como boletines de entrega, con importante trabajo en el laboratorio con asesoramiento del profesor (un modelo clásico que se aproxima mucho a lo que queremos lograr con la metodología presente, incluida una breve coevaluación) a la teoría, que se mueve por los cauces largo tiempo hollados de los exámenes. 
Así, cada parcial, con un peso de 2,5 puntos cada uno (luego entre los parciales se obtiene el $50 \%$ de la nota total) compuesto por un test y dos preguntas de desarrollo, tienen una forma que no difiere demasiado a la que tenían los exámenes que el autor de esta comunicación realizó hace ya décadas en el mismo centro donde ahora imparte clase. Y con respecto a los casos, dado el celo en no perder el precioso tiempo de exposición en aula, el proceso de obtención de nota se resume en un test por cada caso, de 5 preguntas y 0,5 puntos cada uno, a realizar un día concreto, idéntico para todos los grupos, mediante la herramienta de test de la plataforma poliformat.

En este esquema se percibe que mientras las prácticas si se van evaluando con escalas (5 notas para 10 sesiones), con lo que respecta a la teoría, por estar ocupadas las horas de las sesiones por la clase magistral, queda solo resquicio para tres casos pequeños, a realizar en la última media hora de tres sesiones. O, dicho de otra forma, una hora y media dedicada a la evaluación de las 30 horas de aula de teoría.

Resulta claro que no solo "había que llenar" el tiempo que se recuperaba al sacar de la pizarra y trasladar a la plataforma los contenidos teóricos, sino que se presentaba la mejor oportunidad para llevar a cabo esa evaluación continua que desde todos los estamentos nos llega como una realidad. Realidad que, todo hay que decirlo, resulta ilusoria a poco que rasquemos la pintura de la escayola en algunas materias (y si alguien precisa pruebas de ello, solo tiene que visitar los foros extraoficiales de los alumnos, una de las mejores maneras de averiguar la percepción que tienen de nosotros y nuestras clases).

Para llevar esto a cabo, creamos el modelo que presentamos ahora.

\subsection{Evaluación de los grupos FLIP}

De igual modo que en el caso anterior, vamos a empezar con una tabla sintética que, en este caso, desglosaremos con detalle.

Tabla 2. Evaluaciones en grupos FLIP

\begin{tabular}{|l|l|l|l|}
\hline Corresponde a & Tipo & Actos & Peso \\
\hline Práctica & Trabajo académico & 4 & $30 \%(1+1+0,5+0,5)$ \\
\hline Práctica & Coevaluación & 1 & $5 \%(0,5)$ \\
\hline Teoría & Caso de aula & 3 & $15 \%(0,5+0,5+0,5)$ \\
\hline Teoría & $\begin{array}{l}\text { Tarea a realizar en } \\
\text { casa, basada en un } \\
\text { caso }\end{array}$ & 3 & $30 \%(1+1+1)$ \\
\hline Teoría & Caso de aula & 2 & $20 \%(1+1)$ \\
\hline
\end{tabular}

¿Qué cambios se han hecho?

En cuanto a las prácticas, dado que estas, organizadas en grupos de trabajo, se hacen en laboratorio, quedando la memoria a elección de ellos el realizarla allí o, dedicar más tiempo

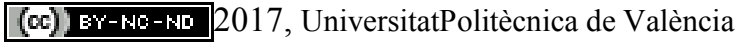


de laboratorio para terminar de forma más cumplida los trabajos y con apoyo de los profesores de prácticas, y terminarlas en casa (de cualquier forma, se entregan mediante una tarea de la plataforma Poliformat), se decidió dejarlas como estaban. El seguimiento permite evaluar el trabajo de los estudiantes no solo a lo largo del tiempo, sino con más cercanía, pues los grupos de prácticas son más reducidos que los de teoría, y además, sus trabajos se realizan en grupos de tres o cuatro alumnos, lo que permite jugar con la tutoría interpares que se produce de forma natural en ellos.

En la teoría, aunque en apariencia no todo se ha cambiado, lo cierto es que la renovación ha sido total. Vamos a ver lo cambios, centrándonos en la evaluación.

Decíamos que en el aula se usaba una escasa hora y media para tres casos, que sumaban 0,5 puntos. Esos casos se evalúan tras un breve debate con un test online. Puede percibirse que el tiempo es en extremo reducido, pero dado que el temario tiene una extensión considerable y hay que verlo todo en clase, poco tiempo resta para las alharacas docentes. El resto de la teoría se evaluaba con dos exámenes parciales que con un peso total de 5 puntos completaba la carga destinada a evaluar la teoría en la nota de la asignatura.

Hemos insistido en el cambio en sí de las clases. Vale la pena detenerse unos instantes en este punto y ver cuál ha sido ese cambio.

En la docencia convencional, la clase de dos horas tiene una pequeña introducción de 10 minutos que ubica al alumno en la materia, haciendo un breve resumen de lo visto, $\mathrm{y}$, salvo cuando hay un caso a ver en aula, el resto del tiempo se dedica a la exposición de los temas de teoría. Si es una de las tres sesiones donde se evalúa con un caso, la última media hora en lugar de emplearla para el desarrollo de teoría, se invierte en unos 20 minutos de debate y diez para la cumplimentación de un test.

En los grupos que siguen el modelo FLIP se decidió emplear cuatro de las cinco sesiones como clase de repaso, de tutoría abierta, y el resto, con un esquema como el siguiente:

- Los primeros diez minutos se dedican a ubicar nuestro punto de mira: no solo donde estamos en el temario, que contenidos son los que deben ver durante la semana en curso (videos, audios, documentos de lectura) y que tareas permanecen abiertas y han de responder si no lo han hecho ya, junto con la previsión de actividades en aula para las siguientes sesiones.

- En la siguiente media hora se hace un repaso de los ejes principales de la teoría. No se trata de volver a contar lo que ya han leído, sino de subrayar lo más importante de ella, donde deben prestar atención y, dado que en la siguiente parte de la sesión se va a trabajar con un caso, se incide en los elementos más relevantes de la teoría para el mismo.

- Durante la siguiente hora y veinte minutos se trabaja un caso. Este puede tener formas muy diversas.

- Pueden ser los tres casos cortos pre-existentes, que mantienen su peso y se desarrollan en la misma fecha que en el resto de los grupos, pero a los que se da más tiempo para el debate, se examinan elementos que puedan aportar luz, se profundiza en las implicaciones que podrían suponerles situaciones similares en 
su vida laboral... Los últimos veinte minutos se evalúa, pero no con un test, pues éste es sustituido por un breve desarrollo.

- Los cinco puntos que sustituyen a los parciales corresponden a casos (inciso: se les ofrece cuando se presenta la asignatura que escojan entre la evaluación convencional o está basada en casos de aula. Generalmente la respuesta es que un $100 \%$ del alumnado escoge la opción propuesta. Salvo este año, pues alumnos procedentes de otros grupos han pedido cursar la asignatura en grupos FLIP al oír hablar del modelo y gustarles más), casos que pueden ser evaluados mediante una tarea o en el aula. Con estos últimos poca diferencia hay sobre los anteriores, salvo que el desarrollo no es tan breve, y se les da treinta minutos para cumplimentarlo. Pero hay algunos que necesitan de bien el manejo de alguna herramienta informática, bien la búsqueda de información por su parte. Estos son los que se evalúan mediante tarea. Una vez visto el caso en el aula, disponen de una semana para entregarla mediante Poliformat.

Otros aspectos a considerar:

- Gracias a la centralización de los materiales que van a usar en el apartado "Contenidos" de Poliformat, algunos casos van apoyados de vídeos documentales que bien les aportan ayuda para preparar el debate, bien les da pistas para su trabajo autónomo al preparar las tareas. Un par de ejemplos significativos serían los documentales '¡COPIAD, MALDITOS!': LOS CAMINOS ALTERNATIVOS AL 'COPYRIGHT' y OJO CON TUS DATOS. En estos casos se habilita también un foro en poliformat para que se pueda discutir en ellos antes y después de la clase.

- Las clases dedicadas a clase de repaso se emplean para apoyar a aquellos alumnos "atascados" en el desarrollo de una tarea, o para aclarar aspectos de teoría (o ampliar para los interesados. Por ejemplo, en los temas de profesionalismo se desarrollan dos modelos y se cita tan solo a un tercero, que aun no está en vigor: ese modelo por petición de algunos alumnos se desarrollo en una de esas clases, pese a que no "va para examen").

- Vemos que se permite un mayor conocimiento del alumno, al trabajar directamente con él. Esto, aunque no se refleja directamente en la nota, permite al docente apoyarle en su proceso de formación de una forma muy directa, dándole consejos de lecturas, respondiendo dudas puntuales que de otra manera no se hubieran planteado, etc.

\section{Resultados}

En el momento de redactar esta comunicación, la asignatura se encuentra en su punto medio del recorrido. Dos elementos cuantitativos podríamos comparar, la asistencia y la nota acumulada. Pero quedaría cojo sin hablar también de un elemento tan importante, aunque cualitativo, que es la opinión expresada por los alumnos.

\subsection{Asistencia}

Sería trampa considerar tan solo las hojas de firmas. Y lo sería, pues hemos tenido sesiones de tutoría donde solo los interesados (aquellos que querían ampliar algo, aquellos con una tarea atascada o aquellos con interés en aclarar algún punto, acuden). El resto, tiene ese

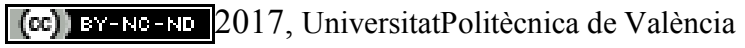


tiempo como apoyo para las actividades de fuera de aula, que son abundantes. (Aunque de hecho un buen grupo de ellos acude a clase y trabaja en el aula con sus portátiles en esas actividades, así, si se encuentran un problema al hacerlas, tienen al profesor para que les apoye). Considerando las clases no de repaso, la asistencia supera en un 5\% al resto de grupos (en la ETSINF se pasa parte de firmas y la asistencia de por sí, es elevada)

\subsection{Nota acumulada}

La nota acumulada es sensiblemente más alta en los grupos FLIP (en torno a un 20\%, aunque no se puede establecer una relación directa por el distinto calendario de evaluaciones, pero si se puede jugar con las proporciones). Aquí hay que ser sincero y dar una explicación que permite una lectura del dato más completa: las tareas entregadas (de momento dos) son revisables si se entregan en un plazo determinado. El profesor las corrige e indica sus fallos. Si deciden rehacerlas, evidentemente su nota sube.

\subsection{Opiniones}

No solo tenemos, como se ha apuntado antes, alumnos que cambian de grupo, sino que los comentarios informales con ellos son realmente gratos. A modo de ejemplo, vemos en la figura siguiente un par de líneas procedentes de un intercambio de correos con una alumna.

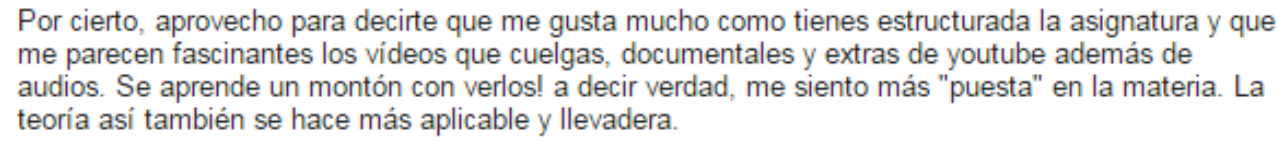

Por cierto, aprovecho para decirte que me gusta mucho como tienes estructurada la asignatura y que me parecen fascinantes los vídeos que cuelgas, documentales y extras de youtube además de audios. Se aprende un montón con verlos! a decir verdad, me siento más "puesta" en la materia. La teoría así también se hace más aplicable y llevadera.

Figura 1. Intercambio epistolar con una alumna, sobre la estructura de los contenidos FLIP

\section{Conclusiones}

En los dos primeros años de docencia FLIP, el problema a batir era el tiempo que el profesor dedicaba a la corrección. Con el modelo actual, donde se han evitado trabajos largos (como en el primer año) y se han reducido el número de casos y tareas, parece que se alcanza el punto de equilibrio.

En las Jornadas de Innovación Docente se escuchó una voz repetida: se decía que las asignaturas con mucha carga teórica no eran susceptibles de seguir un modelo FLIP. Con este trabajo se demuestra que mi creencia en lo contrario no estaba desencaminada. Alumnos con mejores notas, con más interés (de verdad, muy pocas veces me ha sucedido que me pidieran que explicase en clase cosas que seguro que no iban a entrar en la evaluación de la materia, aunque considerase que eran interesantísimas) y alguna que otra felicitación capaz de sonrojar, animan a proseguir con el trabajo.

Por no cerrar el mismo solo con notas positivas, que de todo hay, cabe apuntar un par de elementos que no pueden dejarse de lado: la elaboración de materiales, búsqueda de documentación o vídeos de elementos de actualidad que sirvan para la evaluación de la asignatura, conllevan un tiempo elevado, que nadie se llame a engaño. Por otra parte, también implican una dedicación no puntual, sino continua. Lo que más valoran los 
alumnos es la respuesta inmediata. Las quejas que he tenido que escuchar (a modo de maestro armero, pues eran quejas ajenas a la asignatura) siempre tenían de fondo una constante: "el poco caso que me hacen" o "lo mucho que se retrasa la respuesta de los profesores".

Ambos problemas, es obvio, se traducen en tiempo. Ese tiempo que siempre nos es escaso al docente y que muchas veces no da de sí, por muy bien que creamos gestionarlo.

\section{Referencias}

';Copiad, malditos!': los caminos alternativos al 'copyright' (director: Stéphane M. Grueso). RTVE, 2011. <http://www.rtve.es/television/documentales/copiad-malditos/> [Consultado: 31 de marzo de 2017]

BENNETT B., KERN J., GUDENRATH A., MCINTOSH P (2011). "The Flipped Class Revealed" The Daily Riff.<http://www.thedailyriff.com/articles/the-flipped-class-what-does-a-good-one-looklike-692.php>[Consulta: 31 de marzo de 2017]

BETHANY B. S- (2012) "Flip Your Classroom to Increase Active Learning and Student Engagement" VV.AA. 28th Annual Conference on Distance Teaching \& Learning Wisconsin, EE.UU. University of Wisconsin, 1-5

MARTÍNEZ, A., HERNANDO, A. (2014). "Cómo darle la vuelta al aula: flipped classroom, una metodología para la interacción, la colaboración, el compromiso y la motivación en la clase de ELE" XXV Congreso Internacional de la ASELE. La enseñanza de ELE centrada en el alumno. $<$ http://cvc.cervantes.es/Ensenanza/biblioteca_ele/asele/pdf/25/25_1117.pdf $>$ [Consulta: 31 de marzo de 2017]

MCKEACHIE, W.J., SVINICKI, M.D. (2014).McKeachie'sTeaching Tips: Strategies, Research, and Theory for College and University Teachers, Belmont: Wadsworth

MOWAFY A., KUHN, M., SNOW, T (2013) "Blended learning in highere ducation: Current and future challenges in surveying education in Issues" Educational Research, 23(2): Special Issue, 132150

Ojo con tus datos (Guion: Marisol Soto). RTVE, 2013 $<\mathrm{http}$ //www.rtve.es/alacarta/videos/documentos-tv/documentos-tv-ojo-tus-datos/2270048/> [Consultado: 31 de marzo de 2017]

OLTRA, J.V. (2015) "Diseño de una experiencia de Flip-Teaching para la asignatura Deontología y Profesionalismo a impartir en la Escuela Técnica Superior de Ingeniera Informática de la UPV" INRED $2015 \quad<$ http://ocs.editorial.upv.es/index.php/INRED/INRED2015/paper/view/1646> [Consultado: 31 de marzo de 2017]

OLTRA, J.V. (2016) “ Un año de FLIP: Corrigiendo errores” IN-RED 2016 $<$ http://ocs.editorial.upv.es/index.php/INRED/INRED2016/paper/view/4415> [Consultado: 31 de marzo de 2017]

RIZZO, S. [et al.]. (2015). "Flipped classroom, LCA y materiales de construcción: una experiencia didáctica para una actividad de aprendizaje cooperativa y activa". Jornades sobre Innovació Docent en Arquitectura (JIDA). "III Workshop on Educational Innovation in Architecture (JIDA'15), Barcelona School of Architecture, 25th to 29th May 2015". Barcelona: Grup per a la Innovació i la LogísticaDocent en l'Arquitectura, 2015.<http:/hdl.handle.net/2117/81700> [Consulta: 31 de marzo de 2017]

(cc) EY-NC-ND 2017, UniversitatPolitècnica de València 
ROSHAN S. (2011) "The best way to reacheach student? Private school Math teacher flips learning" The Daily Riff. $<$ http://www.thedailyriff.com/articles/the-best-way-to-reach-each-student-privateschool-flips-learning-547.php> [ Consulta: 31 de marzo de 2017]

SAMS A., BERGMANN J. (2013) "Flip Your Students' Learning" Technology-Rich Learning Vol 70 $\mathrm{n}^{\circ} 6$

SPENCER D., WOLF D.\& SAMS A (2011) "Are you ready to flip?" The Daily Riff. $<$ http://www.thedailyriff.com/articles/are-you-ready-to-flip-691.php $>$ [Consulta: 31 de marzo de 2017]

VASILEVA-STOJANOVSKA T., MALINOVSKI T., DOBRIJOVEVSKI M.V., TRAJKOVIK V. (2015) "Impact of satisfaction, personality and learning style on educational outcomes in a blended learning environment" Learning and Individual Differences $<$ do:10.1016/j.lindif.2015.01.018>[ Consulta: 31 de marzo de 2017] 